\title{
Loss of retinoic acid receptor-related receptor alpha (Rora) promotes the progression of UV-induced CSCC
}

\author{
Guolong Zhang (1)', Guorong Yan (1)', Zhiliang Fu²,3 Yuhao wu', Fei Wu', Zhe Zheng', Shan Fang', Ying Gao',

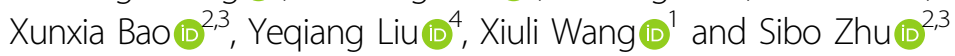

\begin{abstract}
Cutaneous squamous cell carcinoma (CSCC) is prevalent in the world, accounting for a huge part of non-melanoma skin cancer. Most CSCCS are associated with a distinct pre-cancerous lesion, the actinic keratosis (AK). However, the progression trajectory from normal skin to AK and CSCC has not been fully demonstrated yet. To identify genes involved in this progression trajectory and possible therapeutic targets for CSCC, here we constructed a UV-induced CSCC mouse model covering the progression from normal skin to AK to CSCC, which mimicked the solar UV radiation perfectly using the solar-like ratio of UVA and UVB, firstly. Then, transcriptome analysis and a series of bioinformatics analyses and cell experiments proved that Rora is a key transcript factor during CSCC progression. Rora could downregulate the expressions of S100a9 and Sprr2f in CSCC cells, which can inhibit the proliferation and migration in CSCC cells, but not the normal keratinocyte. Finally, further animal experiments confirmed the inhibitory effect of cSCC growth by Rora in vivo. Our findings showed that Rora would serve as a potential novel target for CSCC, which will facilitate the treatment of CSCC in the future.
\end{abstract}

\section{Introduction}

Cutaneous squamous cell carcinoma ( $\mathrm{CSCC}$ ) is the second most common non-melanoma skin cancer with 1.8 million incidences in a global context in $2017^{1}$. The progressions of cSCC from normal skin (NS) are determined by genetic susceptibility and environment risk factors including ultraviolet radiation, carcinogenic chemicals, and immunosuppressive drugs ${ }^{2}$. Although CSCC is characterized by a relatively low risk of metastasis and with long-term survival after treatment, there were still

\footnotetext{
Correspondence: Yeqiang Liu (lyqdoctor@163.com) or

Xiuli Wang (wangxiuli_1400023@tongji.edu.cn) or

Sibo Zhu (sibozhu@fudan.edu.cn)

'Institute of Photomedicine, Shanghai Skin Disease Hospital, Tongji University School of Medicine, Shanghai 200443, China

${ }^{2}$ State Key Laboratory of Genetic Engineering, School of Life Sciences, Fudan University, Shanghai 200438, China

Full list of author information is available at the end of the article These authors contributed equally: Guolong Zhang, Guorong Yan Edited by D. Aberdam
}

over $2.1 \%$ of cSCC patients that developed into lymphatic metastases and 1.5\% deceased in 2012 in the US white population $^{3}$. Furthermore, the ageing and growing population have led to an increasing incidence of CSCC, especially for the aged. Extensive researches have been carried out to uncover the pathogenesis behind CSCC from various omics aspects including genomics ${ }^{4}$, transcriptomics $^{5}$, proteomics $^{6}$, and epigenetics ${ }^{7}$.

Fourteen susceptive loci and 13 candidate genes including DEF8, IRF4, MC1R, etc. associated with high cSCC risk were identified by genome-wide association studies in different populations ${ }^{8-10}$. Except for the susceptive genes, high aberrated genes were also investigated in cSCC patients by whole-exome sequencing technology. In specific, NOTCH1/2, TP53, CDKN2A, HRAS, etc. held a very high mutated rate ${ }^{11-14}$. What is more, the expression profile of cSCC has been quantified by either gene expression chips or RNA-sequencing (RNA-seq) technology, and a large number of differentially expressed

\section{(c) The Author(s) 2021}

(c) (i) Open Access This article is licensed under a Creative Commons Attribution 4.0 International License, which permits use, sharing, adaptation, distribution and reproduction c. in any medium or format, as long as you give appropriate credit to the original author(s) and the source, provide a link to the Creative Commons license, and indicate if changes were made. The images or other third party material in this article are included in the article's Creative Commons license, unless indicated otherwise in a credit line to the material. If material is not included in the article's Creative Commons license and your intended use is not permitted by statutory regulation or exceeds the permitted use, you will need to obtain permission directly from the copyright holder. To view a copy of this license, visit http://creativecommons.org/licenses/by/4.0/. 
genes (DEGs) were detected ${ }^{5,15}$. As more and more focuses were received on the process of post-transcription and histone modification, the dissection of pathogenesis behind CSCC are spreading from genetics to epigenetics field $^{16,17}$. However, although many significant features were identified enriched or suppressed in $\mathrm{CSCC}$, the key factors underlying the progression of NS to cSCC remains to be elucidated.

Actinic keratoses (AK), histologically characterized by epidermal thickening and spinous layer atypia, is considered to be a precancerous lesion of $\mathrm{CSCC}^{18}$. The hazard of progression of AK to CSCC was estimated to be $0.6 \%$ within one year, then increased to $2.57 \%$ within 4 years ${ }^{19}$. Although, the estimated progression rate was low, $~ 65 \%$ cSCCs were diagnosed clinically as AKs, previously ${ }^{19}$. Thus, it is of great importance to uncover the mechanism involved in the progression progress from NS to AK, then to cSCC. On the contrary, it is more difficult to identify the driver gene than other malignancies, as $\mathrm{CSCC}$ is the most highly mutated malignancy with a complex genetic background ${ }^{20}$. Therefore, a functional-based biomarker is more essential to identify the mechanism. Lambert et al. ${ }^{21}$ found that the mitogen-activated protein kinase pathway may be important for the transition of AK to CSCC by comparing the transcriptome profiling of $10 \mathrm{AKs}$ and 30 cSCCs. Similarly, Chitsazzadeh et al. ${ }^{22}$ detected a series of transcription factors (TFs) such as ETS2, SP1, FREAC2, and AP1 which should be responsible for the gene expression changes observed during the stage of AK and cSCC development by a cross-species identification strategy. However, the identified TFs either lacked necessary validation in terms of pathway or function nor explain the whole process from NS to $\mathrm{cSCC}$ via AK stage.

In the present study, we aim to identify transcriptomic markers playing critical roles in the progression of CSCC from NS and validate the AK as a transitional state. We firstly established a UVR-induced cSCC model using SKH-1 hairless mouse. Then, the transcriptome profiles among NS, AK, and CSCC tissues were investigated. We further constructed a pseudo-time trajectory to validate the NS-AK-cSCC path route, through which we found that loss of retinoic acid receptor-related receptor alpha (Ror $\alpha$ ) was involved in the process. Further wet-lab assays and animal experiments were carried out to understand the function of Rora and possible down-stream targets. This study unraveled the pathogenesis progression of UVR-induced cSCC, explained Ror $\alpha$ based function in the process, and enlighten a promising therapy of $\mathrm{CSCC}$.

\section{Materials and methods \\ UVR-induced CSCC mouse model construction and tissue collection}

Eight 7-month-old female hairless SKH-1 mice purchased from Shanghai Laboratory Animal Center
(Shanghai, China) were randomly and equally divided into two groups: ultraviolet free (UVF) and ultraviolet radiation (UVR) group. The UVR group was used to model UVR-induced cSCC under controlled conditions. In detail, mice in the UVR group were irradiated with ultraviolet rays from the SUV1000 daylight ultraviolet simulator (SIGMA, Shanghai, China) with built-in UVA and UVB filters. Preliminary experiments found that the initial minimal erythema dose (MED) was $160 \mathrm{~mJ} / \mathrm{cm}^{2}$ for UVB and $2520 \mathrm{~mJ} / \mathrm{cm}^{2}$ for $\mathrm{UVA}^{23-25}$. The UV irradiation was given four times every week for 28 weeks. As the mice in the UVR group have an increased tolerance to ultraviolet radiation as the experiment progresses by, the light dose needs to be enhanced till 1.625 MED for the end of 20 weeks. At the end of the experiment, the cumulative dose for UVA and UVB were 242.91 and $26.99 \mathrm{~J} / \mathrm{cm}^{2}$, respectively. The tumor numbers and diameters in the UVR group were recorded weekly. After 28 weeks, all mice were euthanized by carbon dioxide inhalation.

All skin tissues were isolated from the back of mice (Fig. 1a). In specific, one piece of NS tissue was isolated from each mouse in the UVF group (NS-1, NS-2, NS-3, NS-4). In the UVR group, one piece of NS and three skin lesion tissues, including actinic keratosis grade 1 (AK-1), AK grade 2 (AK-2), and CSCC were collected. These tissues were validated by a pathologist with hematoxylineosin (HE) staining and defined as NS, AK-1, AK-2, and cSCC. For HE staining, part of each tissue was immediately fixed with $4 \%$ formaldehyde solution. For transcriptomic analyses, the rest part was collected in TRIZOL (Sigma, USA) solution for RNA extraction.

\section{Patient sample collection}

Primary cSCCs and patient-matched normal adjacent samples for immunohistochemistry (IHC) staining were obtained from consecutive cSCC patients, all of whom were immunotherapy-naive. All diagnoses of $\mathrm{cSCC}$ were verified histologically by a board-certified dermatopathologist. The study was approved by the Institutional Review Board of Shanghai Skin Disease Hospital.

\section{mRNA library preparation for sequencing}

Total RNA was extracted using TRIZOL reagent as per manufacturer's instruction. cDNA libraries were prepared by Nextera sample preparation kit (Illumina, USA). Index labeled libraries sized at 300-450 bp length were recovered by using Ampure XP Beads (Beckman Coulter, USA). All libraries were quantified using 2100 Bioanalyzer and pooled as 1:1 at $2 \mathrm{nM}$ for 150 paired-end modes and were further sequenced by NextSeq 500 (Illumina, USA).

\section{RNA-seq analysis}

The quality of raw reads was evaluated by using FastQC $(\mathrm{v} 0.11 .5)^{26}$. Then, low-quality reads were filtered by 


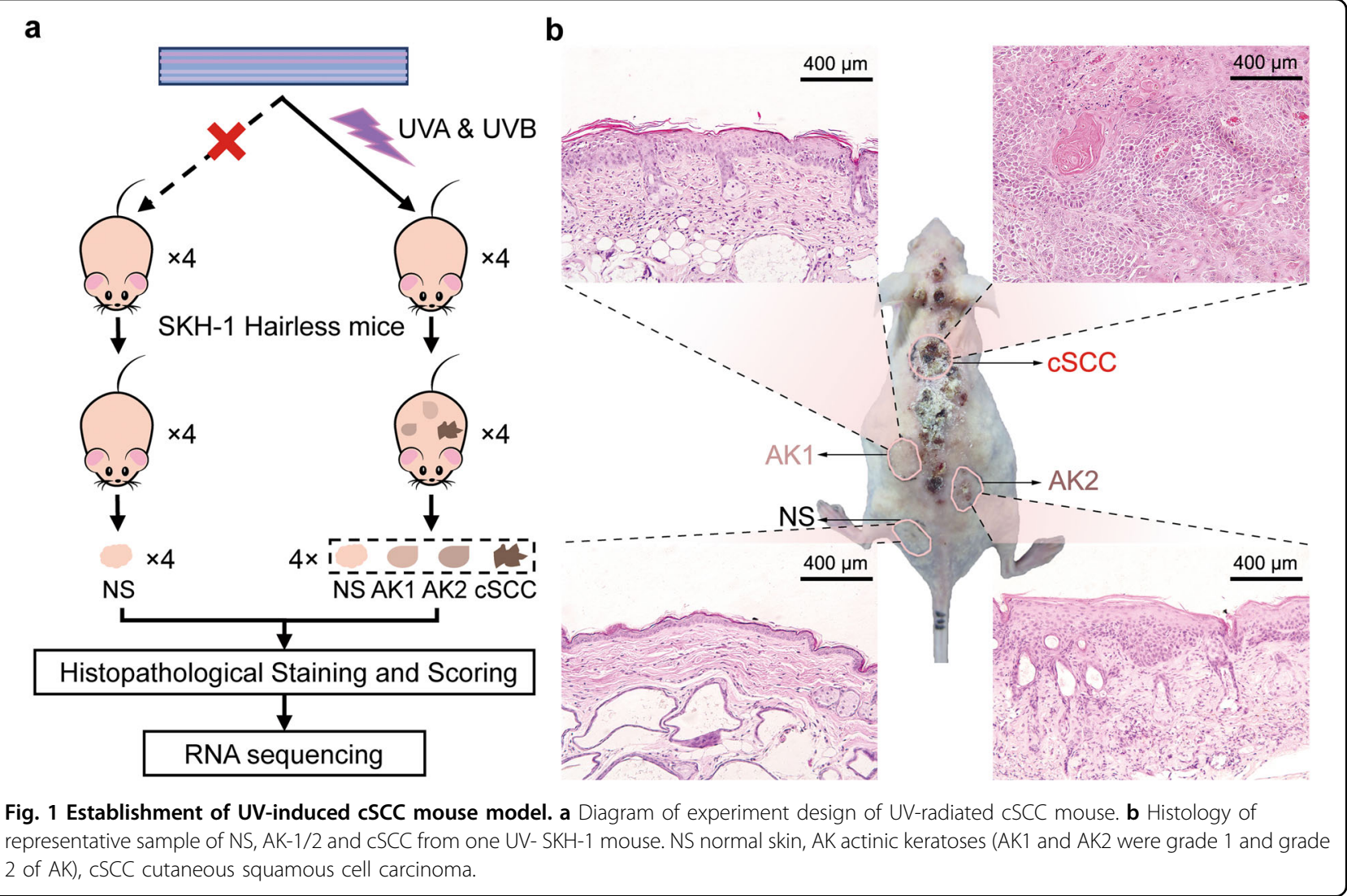

Trimmomatic $(\mathrm{v} 0.36)^{27}$. Reads with an average Phred quality score $<20$ were discarded after trimming. All the remaining qualified reads were aligned to the mouse reference genome (GRCm38) using HISAT2 ${ }^{28}$. Then, StringTie was employed for gene expression quantification to build an expression matrix ${ }^{29}$.

Data pre-processing, Pearson correlation, and hierarchical clustering analysis calculated by ward method were performed with base and stat R-packages based on $\mathrm{R}$ software (v3.6.2). R-Packages including limma, edgeR, and ggplot2, ComplexHeatmap were used for data normalization and visualization. The identification of DEGs was performed by calculating fold changes (FC) and average expression differences using the normalized value of each gene, and statistical significance was tested by Student's ttest. Then, DEGs were determined by the threshold of fold change $>1.5$ or $<0.67$, and $p$ value $<0.05$.

\section{Gene set variation analysis}

In order to further identify pathways enriched in different groups, gene set variation analysis (GSVA) using gene set file of biological processes from the Molecular Signatures Database (c5.bp.v7.1.symbols.gmt, MSigDB v7.1 from http://bioinf.wehi.edu.au/software/MSigDB/) was performed as implemented in the GSVA ${ }^{30} \mathrm{R}$-package (v1.34.0, default parameters), in which the gene-bysample matrix is converted into a pathway-by-sample matrix. Then, the differentially expressed pathway was identified by limma R-package with the same threshold in DEGs identification.

\section{Immune microenvironment analyses}

In order to investigate the immune microenvironment (IME) change during cSCC progression, we next inferred the compositions of immune and stroma cells using enrichment score-based algorithm xCell from RNA-seq data $^{31}$. Briefly, enrichment scores of 36 immune cells were obtained from the gene expression matrix of all 20 samples using immunedeconv R-package. Then, the $\mathrm{T}$ cell subset, monocyte, and neutrophil were generated by summation of the scores in each sample ${ }^{32}$. Heatmap was generated based on pathological stages. To further understand the relationship between the pathological stage and the IME change, we employed univariate regression analysis between TPS (tumor progression stage) and cell subsets fraction.

\section{Pseudo-time trajectory construction}

To investigate the progression trajectory of $\mathrm{cSCC}$, we used minimal spanning tree algorithm-based Monocle (v2) 
R-package to perform a pseudo-time analysis. We assumed that the trajectory had a tree structure, with a root state of related NS, and a leave state of tumor cells. Samples were colored as their pathological classifications.

\section{Transcription factor co-regulatory network}

Gene co-expression networks were firstly built according to the normalized gene expression values. The Pearson correlation coefficients of genes were obtained as the gene-to-gene co-expression adjacency matrix. We further applied transcription factors as core genes (AnimalTFDB, $\mathrm{v} 3.0)^{33}$ and removed nodes with absolute correlation coefficient $<0.5$, false discovery rate $>0.05$ or number of edges $<5$, to draw a transcription factor co-regulatory network CytoScape (v3.7.3).

External validation of Rora expression and survival analysis

Ror $\alpha$ expression was investigated by immunochemistry staining and single-cell datasets. The pathology-stained micrographs of Ror $\alpha$ from normal and cSCC tissues were downloaded from the Human Protein Atlas (HPA) database. The optical density (OD) of Ror $\alpha$ between NS tissue and cSCC pathological was compared by 'Image J' software. Furthermore, large-scale single-cell RNAsequencing (scRNA-seq) data from human cSCCs and matched NS with 48,164 cells (GSE144240) was obtained from Gene Expression Omnibus (GEO) database, which enabled us to analyze the Ror $\alpha$ expression at singlekeratinocyte level. As cSCC database with clinic information was unavailable and head and neck squamous cell carcinoma (HNSCC) is often used as an alternative of cSCC in most studies ${ }^{22,34}$. Thus, we used HNSCC to explore the association between Ror $\alpha$ and survival. TCGA based survival data were downloaded from OncoLnc (http://www.oncolnc.org/). The analysis was performed with survival and survminer R-packages.

\section{IHC staining of CSCC patients}

cSCCs and patient-matched normal adjacent samples were fixed in 10\% neutral buffered formalin, dehydrated with gradient ethanol, hyalinized in xylene, embedded in paraffin, and made into sections. Sections $(5 \mu \mathrm{m})$ of paraffin-embedded tissue were deparaffinized, dehydrated with gradient ethanol, and rinsed with tap water before antigen repair and serum blocking. Next, the primary anti- Ror $\alpha$ antibody (Abcam: ab60134, $0.5-10 \mu \mathrm{g} / \mathrm{ml}$ ) was added for overnight reaction at $4{ }^{\circ} \mathrm{C}$, followed by the addition of secondary antibody for $1 \mathrm{~h}$ of incubation. 3, 3'-Diaminobenzidine tetrahydrochloride solution was used for $15 \mathrm{~min}$ of coloration, followed by counterstaining with hematoxylin, dehydration with gradient ethanol, hyalinization with xylene, mounting with neutral resin, and observation under a microscope.

\section{Interference, activation assay, and qRT-PCR of RORA, S100a9, and Sprr2f}

Human-derived cell line A431, $\mathrm{HaCaT}$, and mouse cell line PECA from the National Infrastructure of Cell Line Resource were seeded in cell culture dishes and cultured for 3 days till confluency at 90\%. Since Ror $\alpha$ expressed lowly in cancer cells (data not shown), we employed Ror $\alpha$ agonist only to understand its function. Ror $\alpha$ agonist SR1078 (Selleck, USA) and small interfering RNA (siRNA)-Hilymax complex (S100a9 and Sprr2f) were added to the cells, incubated at $37^{\circ} \mathrm{C}$ for $48 \mathrm{~h}$.

After reverse transcription of RNAs from cells, a quantitative real-time PCR (qRT-PCR) reaction system was setup. SYBR green dye (Takara) was applied in the PCR reaction. The $\mathrm{Ct}$ values of the target genes S100a9, Sprr2f, and the housekeeping gene GAPDH were obtained, and the $2^{-\Delta \Delta} \mathrm{Ct}$ algorithm was used for quantitative analysis. Primer and siRNA information were presented in Table 1.

\section{CCK-8 assay, wound-healing assay, transwell assay, and apoptosis assay}

For Cell Counting Kit-8 (CCK-8) (Dojindo, Japan) assay, after cells were activated or interfered for 24 and $48 \mathrm{~h}$, $10 \mu \mathrm{l}$ CCK-8 was added to each well. OD value of each well was measured at $450 \mathrm{~nm}$ using a microplate reader.

Table 1 Primer and siRNA information. Design of qRTPCR primers and siRNAs.

\begin{tabular}{ll}
\hline Primer & Sequence (5'-3') $^{\prime}$ \\
\hline Homo-S100a9-F & AACATAGAGACCATCATCAACACC \\
Homo-S100a9-R & GGTCCTCCATGATGTGTTCTATG \\
Mouse-S100a9-F & TCAATACTCTAGGAAGGAAGGACA \\
Mouse-S100a9-R & AGCTGATTGTCCTGGTTTGT \\
Homo-Sprr2f-F & CCTGCCCACCATCAAAGT \\
Homo-Sprr2f-R & CTTGCTCTTGGGTGGACA \\
Mouse-Sprr2f-F & TGAGCCTTGTCCTCCTCCA \\
Mouse-Sprr2f-R & TCTTGGGTGGGCACTTCTG \\
Homo-GAPDH-F & GAGTCCACTGGCGTCTTCAC \\
Homo-GAPDH-R & ATCTTGAGGCTGTTGTCATACTTCT \\
Mouse-GAPDH-F & TGACCTCAACTACATGGTCTACA \\
Mouse-GAPDH-R & CTTCCATTCTCGGCCTTG \\
Sprr2f-215-siRNA sense & GCAGAAAUGUCCUCCUGUGTT \\
Sprr2f-215-siRNA antisense & CACAGGAGGACAUUUCUGCTT \\
S100a9-267-siRNA sense & GCUUCGAGGAGUUCAUCAUTT \\
S100a9-267-siRNA antisense & AUGAUGAACUCCUCGAAGCTT \\
\hline
\end{tabular}

$F$ forward, $R$ reverse, si small interfering. 
For the wound-healing assay, after the cells reached $90 \%$ confluency, a pipette tip was used to scratch a line on the cell layer. The picture of each wound was recorded by microscope after 0 and $24 \mathrm{~h}$. Image J software was used to calculate the mean value of the distance of each wound.

For transwell assay, cells were seed at upper microholed wells with Matrigel plated containing serum-free RPMI 1640 medium. The wells were placed into the lower well containing $500 \mu \mathrm{l}$ of complete medium (RPMI 1640 and 10\% fetal bovine serum). After incubation at $37^{\circ} \mathrm{C}$ for $48 \mathrm{~h}$, cells were gently removed in the upper wells with a cotton swab. The cells in the lower chamber were fixed with $5 \%$ glutaraldehyde for $10 \mathrm{~min}$ and stained with $1 \%$ crystal violet dye in $2 \%$ ethanol at room temperature for $20 \mathrm{~min}$, then photographed and counted by Image J.

For apoptosis assay, cells were resuspended cells in fixation buffer and stained with Annexin V-FITC and Propidium Iodide solutions respectively for $30 \mathrm{~min}$ on ice. Then cells were then analyzed by flow cytometry (BD Biosciences, USA) with FITC and PI channel.

\section{Animal experiment}

To investigate the effect of SR1078 on cSCC growth in vivo, we conducted an animal experiment. Firstly, 10 eight-week-old SKH-1 mice were randomly divided into two groups ( $n=5$ per group). The injected dose of SR1078 was $20 \mathrm{mg} / \mathrm{kg}$, and the weight of each mouse was estimated to an average of $25 \mathrm{~g}$. SR1078 was dissolved in the solution including $10 \%$ dimethyl sulfoxide (DMSO, Selleck), 40\% PEG300 (Selleck), 5\% Tween80 (Selleck) and $45 \% \mathrm{ddH}_{2} \mathrm{O}$. The agonist group and control group were injected intraperitoneally with $150 \mu \mathrm{l}$ SR1078 solution and DMSO solution, respectively. In specific, three times injection in a week was performed before cSCC cell inoculation. Then, XL50 cSCC cells from China Center for Type Culture Collection (Wuhan, China, $5 \times 10^{6}$ ) and NIH/3T3 cells (mouse embryonic fibroblast, $1 \times 10^{6}$ ) were injected subcutaneously into the backs of mice to establish a cSCC mouse model. After incubation, mice were injected intraperitoneally with SR1078 or DMSO solution daily. Tumor volume was measured every 2 days and calculated as length $\times$ width ${ }^{2} \times 0.5\left(\mathrm{~mm}^{3}\right)$. Eighteen days post-injection, the tumor was harvested for cell proliferation evaluation by IHC of Ki67 expression (MA5-14520, Thermo Fisher Scientific) following the guideline.

\section{Results}

\section{Mouse model and samples collection}

In order to simulate sun exposure accurately, we employed solar simulators containing UVA and UVB to establish cSCC mouse model. Eight SHK-1 Hairless mice were used for the present study, among which four mice were in the UVF group and four were in the UVR group (Fig. 1a). Four NS tissue samples were isolated from the UVF group. After mice developed into cSCC, cSCC tumor samples and matched NS and AK (grade 1 (AK1) and grade 2 (AK2)) samples were isolated from the UVR group (Fig. 1a). The pathological classification and the tumor pathological scoring (TPS) of tissues were also presented (Table 2). All experiment samples were histologically validated. Representative samples regarding NS, AK1, AK2, and CSCC were histologically imaged (Fig. 1b). NS samples showed a uniform distribution of keratinocytes, while AK1 and AK2 exhibited a histological phenotype of UV damage including elastosis, fibrosis, and thicker epidermis. Compared with the former, the tumor cells in CSCC distributed in nests with large deeply staining nuclei, and also with many karyokinetic and acantholytic cells.

\section{Differences among NS, AK, and CSCC}

RNA-seq was performed on NextSeq 500 with a total of 70 million reads generated. Unsupervised hierarchical clustering analysis using all genes showed that the NS and AK were closer compared to the cSCC cluster, showing a transcriptomic similarity over the NS and AK groups (Fig. 2a). A similar pattern was also observed in the principal component analysis (PCA) using all genes (Fig. S1). Specifically, there was no clearly different gene expression between AK1 and AK2. After performing DEGs identification, a total of 2022, 2031, and 1617 DEGs were identified in the comparisons of AK vs. NS, CSCC vs. $\mathrm{NS}$, and $\mathrm{cSCC}$ vs. AK, respectively. The top 10 significantly up- and downregulated genes were highlighted (Fig. 2b). In general, genes such as S100 family, Sprr family, Cxcl family, Ccl family, and Mmp family members were significantly upregulated in cSCC (Fig. 2b).

Next, we used GSVA approach to identified differentially expressed pathways among NS, AK, and cSCC based on gene set file of biological processes from the Molecular Signatures Database. A total of 70, 67, and 76 differentially expressed pathways were identified among each comparison (Fig. 2c). Compared with NS, $\mathrm{CD}^{+} / \mathrm{CD} 25^{+} \mathrm{T}$ proliferation, IFN- $\alpha$, IFN- $\beta$, TLR7, and IL23 pathways were significantly upregulated, while chemokine secretion, Apoptotic signaling, and negatively modulate $\mathrm{T}$ cell proliferation pathways were upregulated in AK. In the comparison between cSCC and NS, VEGF receptor signaling, mast cell chemotaxis, response to hypoxia, megakaryocyte differentiation TLR2, and T cell apoptosis pathways were significantly increased. Finally, regarding the comparison between $\mathrm{cSCC}$ and $\mathrm{AK}$, the increased pathways of programmed cell death, mast cell chemotaxis, response to hypoxia and fibroblast migration were observed, and IFN- 
Table 2 Tumor pathological classification and scoring of mice skin tissues.

\begin{tabular}{|c|c|c|c|c|}
\hline Groups & Subject & Sample name & Pathology & Pathological scoring (0-5) \\
\hline \multirow[t]{4}{*}{ Ultraviolet free (UVF) } & 1 & $N C-1$ & NS-1 & 0 \\
\hline & 2 & $N C-2$ & NS-1 & 0 \\
\hline & 3 & NC-3 & NS-1 & 0 \\
\hline & 4 & NC-4 & NS-1 & 0 \\
\hline \multirow[t]{16}{*}{ Ultraviolet radiation (UVR) } & 5 & $1-N C$ & NS-2 & 0 \\
\hline & & $1-A K-1$ & Grade 1 papilloma (G1P) & 1 \\
\hline & & $1-\mathrm{AK}-2$ & Grade 2 papilloma (G2P) & 2 \\
\hline & & $1-\mathrm{cSCC}$ & $\operatorname{CSCC}$ & 4 \\
\hline & 6 & $2-N C$ & NS-2 & 0 \\
\hline & & 2-AK-1 & Grade 1 papilloma (G1P) & 1 \\
\hline & & $2-A K-2$ & Grade 2 papilloma (G2P) & 2 \\
\hline & & $2-\operatorname{cscC}$ & $\mathrm{CSCC}$ & 5 \\
\hline & 7 & 3-NC & NS-2 & 0 \\
\hline & & 3-AK-1 & Grade 1 papilloma (G1P) & 1 \\
\hline & & 3-AK-2 & Grade 3 papilloma (G3P) & 3 \\
\hline & & $3-\operatorname{cscC}$ & $\mathrm{CSCC}$ & 4 \\
\hline & 8 & $4-N C$ & NS-2 & 0 \\
\hline & & 4-AK-1 & Grade 1 papilloma (G1P) & 1 \\
\hline & & $4-A K-2$ & Grade 3 papilloma (G3P) & 3 \\
\hline & & $4-\mathrm{CSCC}$ & cSCC & 4 \\
\hline
\end{tabular}

$\beta$, IFN- $\gamma, \mathrm{B}$ cell differentiation, and lymphocyte migration pathways and etc. were downregulated in CSCC (Fig. 2c).

As most differentially expressed pathways were immune-related, we next sought to observe the change of immune microenvironment across NS, AK, and $\mathrm{cSCC}$ by RNA-seq deconvolution. The heatmap of the relative expression of immune cells is shown in Fig. S2a. Endothelial cells, cancer-associated fibroblast, natural killer, and $\mathrm{CD} 4^{+} \mathrm{T}$ helper1 (Th1) were hyperactivated in the cSCC group. Regulatory $\mathrm{T}$ cells and Granulocytemonocyte progenitor hold the highest expression in the AK group. The highest microenvironment score and stroma score were observed in cSCC group (Fig. S2b). The immune cells composition regarding sample types were presented in Fig. S2c, d, e. The regression analysis showed that endothelial cell and neutrophil are positively correlated with TPS, indicating an extensive increase of angiogenesis and neutrophil accumulation during cSCC progression (Fig. S2f, g).

\section{Rora is inhibited in CSCC}

The cSCC model was generated by irradiation of UVR from NS. The undetermined tumorigenesis process is intriguing and prompts us to further explore the progression trajectory. The gene expression profile of different stages allowed us to reconstruct the trajectory using. The graph showed a linear progression trajectory, which was fitted to the UVR-induced pathology stage very well (Fig. 3a). The solid black line represented the main route of the minimal spanning tree constructed, which exhibited the backbone and order of the cSCC development along a pseudo-temporal continuum. The trajectory clearly showed the tree starts from NS, goes through AK and ends with cSCCs (Fig. 3b). Then, we further constructed a co-expressed regulatory network between TFs and DEGs, which was involved in the trajectory. The TFs, including Rora and POU domain, class 2, transcription factor 3 (Pou2f3) were found to be involved in the cSCC progression, which co-expressed with S100a9 (a member from the S100 family) and Sprr2f (a member from the Sprr family) (Fig. 3c). In particular, S100a9 and Sprr2f were significantly upregulated in cSCC tissues from the mouse model $(p<0.05$, Fig. S3). Compared with NS, Sprr2f were also significantly upregulated in AK $(p<0.05)$, but no significant up-regulation of S100a9 was observed in AK. In the trajectory of NS-AK-cSCC, the expression of Rora showed a continual decreasing trend in terms of the pathology stage, especially in cSCC (Fig. 3d). 


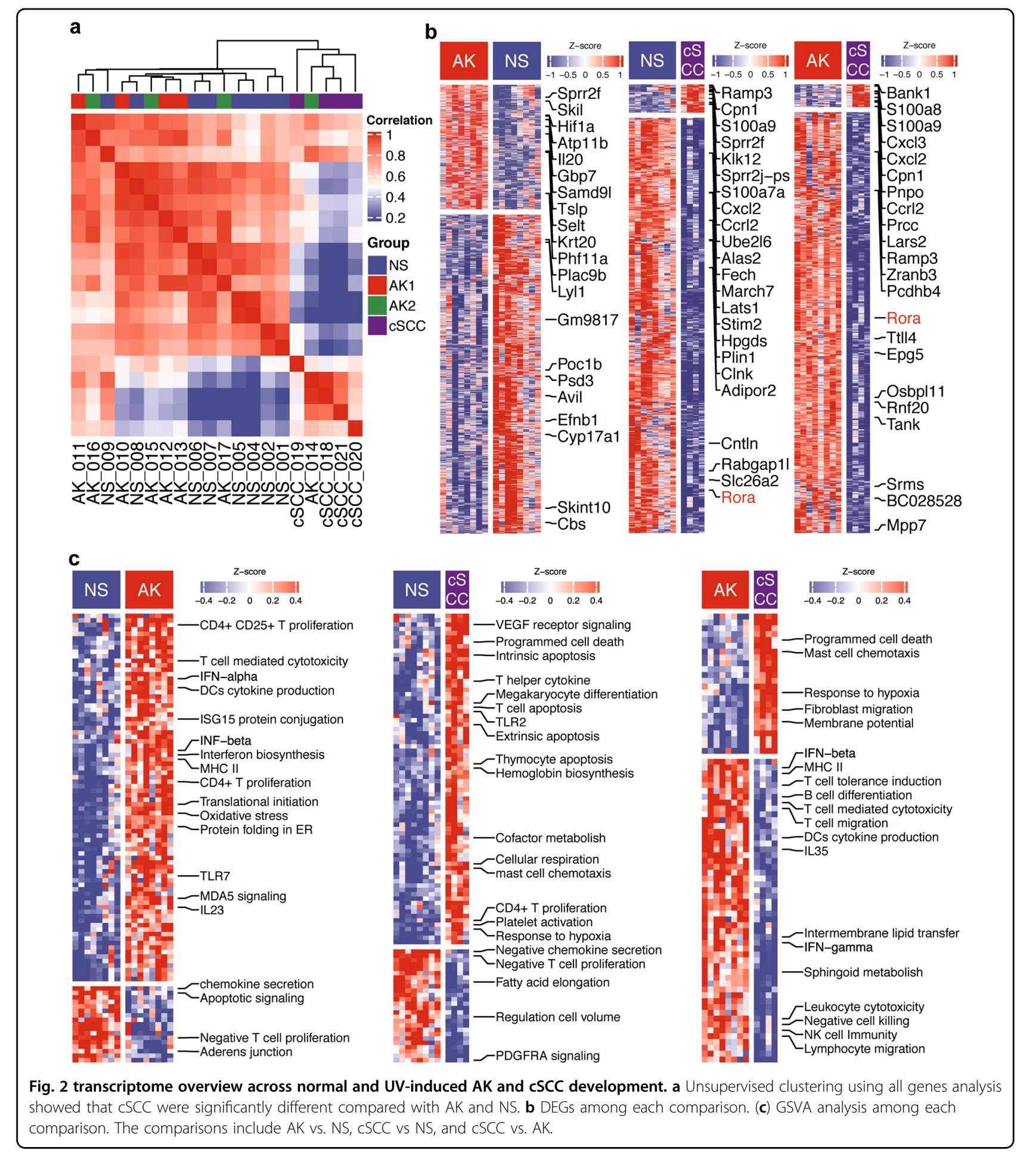

In order to further validate this result, we re-analyzed the histological staining of Rora between NS tissue (five samples) and cSCC tissue (fifteen samples) from the Human Protein Atlas database. A lower relative density of Ror $\alpha$ was observed in $\operatorname{cSCC}(p<0.01$, Fig. 4a), indicating that the expression level of Ror $\alpha$ in cSCC is significantly lower than that of NS, which is in accordance with our result. In addition, as a recent cSCC-related single-cell transcriptomic data with 48,164 cells was released (GSE144240), we further investigate the Ror $\alpha$ expression of skin epithelial cells, from healthy and cSCC tissues. As the violin chart showed, Ror $\alpha$ was significantly 


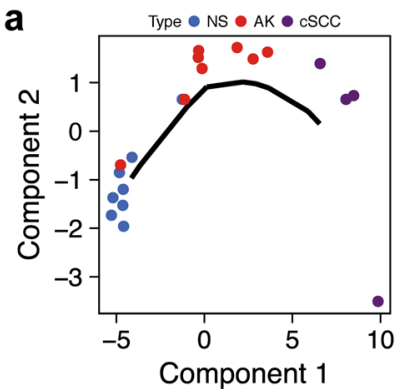

b
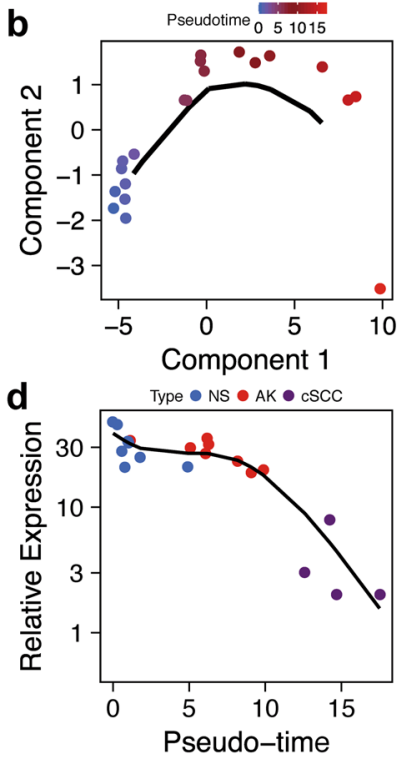

C

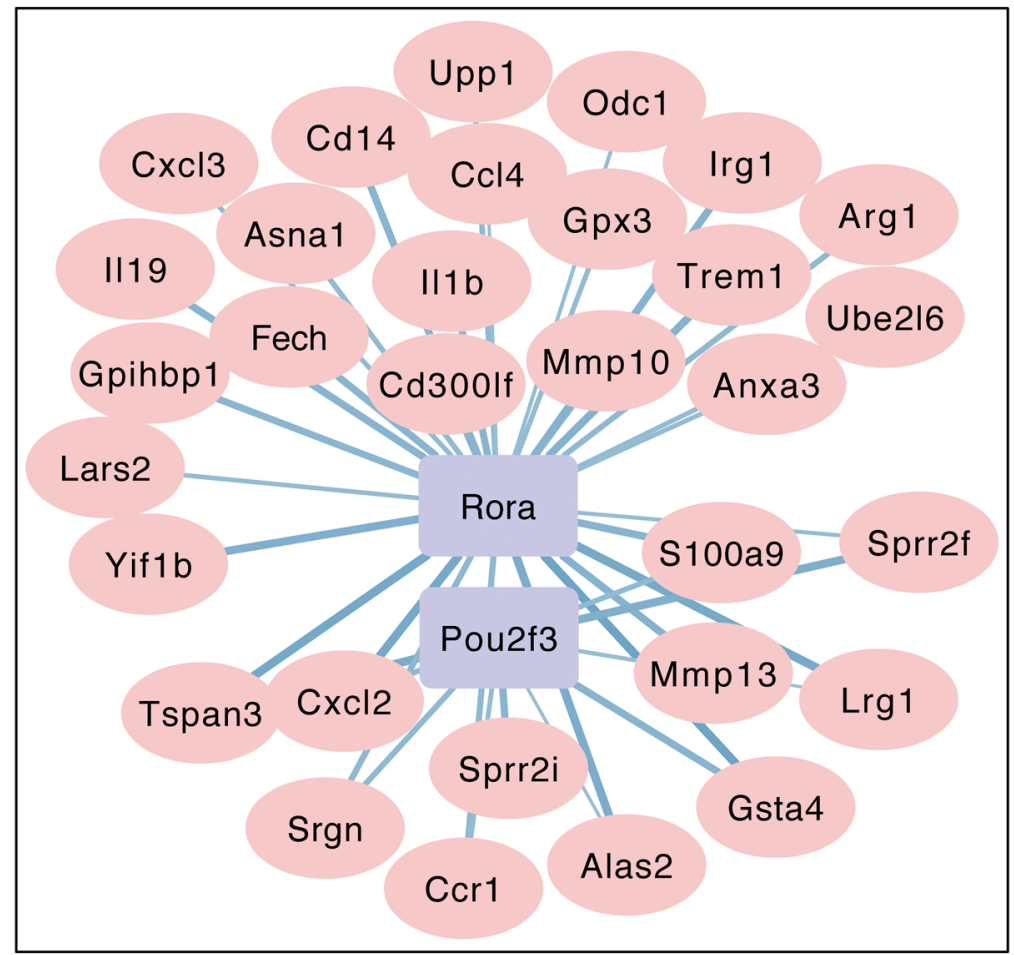

Fig. 3 Reconstructed progression trajectory of UV-induced cSCC are regulated by Rora. a Distribution of NS, AK, and CSCC samples in the pseudo-time trajectory analysis. b Samples were highlighted by pseudo-time in the trajectory, which showed a progression from NS to AK then cSCC. c Co-expressed network calculated between TFs and genes among the CSCC progression-driven genes. Purple rectangle nodes represent TFs and plink elliptic nodes represent genes. $\mathbf{d}$ Rora expression with regards to the trajectory pseudo-time. Rora expression was significantly downregulated in cSCC group.

downregulated in cSCC keratinocytes. (Fig. 4b, c). Furthermore, we calculated the percentage of cells with Ror $\alpha$ $>1$ count, that more Ror $\alpha$ positive cells were exhibited in normal $(72.49 \%)$ keratinocytes than that in the tumor (33.15\%) cells. In summary, we solidly showed that Ror was significantly downregulated in $\mathrm{CSCC}$, which was inhibited during CSCC progression.

IHC staining was performed on the cSCC tissue and NS tissue in CSCC patients. The results showed that Ror highly expressed in the nucleus of keratinocytes in NS, while the expression level in cSCC tissues is significantly reduced (Fig. 4d, e). In order to examine the effect of Ror $\alpha$ on survival time, we employed HNSCC data, which was similar to cSCC. The top 25\% of high and low Rora expression level patients were used for generating a 5-year Kaplan-Meier survival curve. It was found that the patients with high expression of Ror $\alpha$ achieved a better survival prognosis $(p<0.05$, Fig. S4). To conclude, we validated the lower expression of Ror $\alpha$ in both human
CSCC, and the survival results suggested that Ror $\alpha$ plays an important role for patients' survive.

\section{Rora activation inhibits CSCC cell proliferation and migration}

To evaluate the role of Roro in terms of the proliferation and migration ability we performed mouse and human cell-line based assays. As S100a9 and Sprr2f were predicted as genes that are regulated by Ror $\alpha$, we further investigated the expression of these two genes by giving Rora activator in cell lines. In general, S100a9 and Sprr2f were potentially downregulated in cSCC cells (PECA and A431) after being treated with Ror $\alpha$ agonist SR1078. S100a9 was downregulated in both PECA and A431 cells $(p<0.001)$, while extremely downregulation of Sprr2f was observed in $\mathrm{A} 431$ and $\mathrm{HaCaT}$ cells $(p<0.001)$. In the meanwhile, S100a9 expression was slightly upregulated in the $\mathrm{HaCaT}$ cell $(p<0.05)$ (Fig. 5a). Ror $\alpha$ agonist inhibited cell proliferation after stimulating all three types of cells 


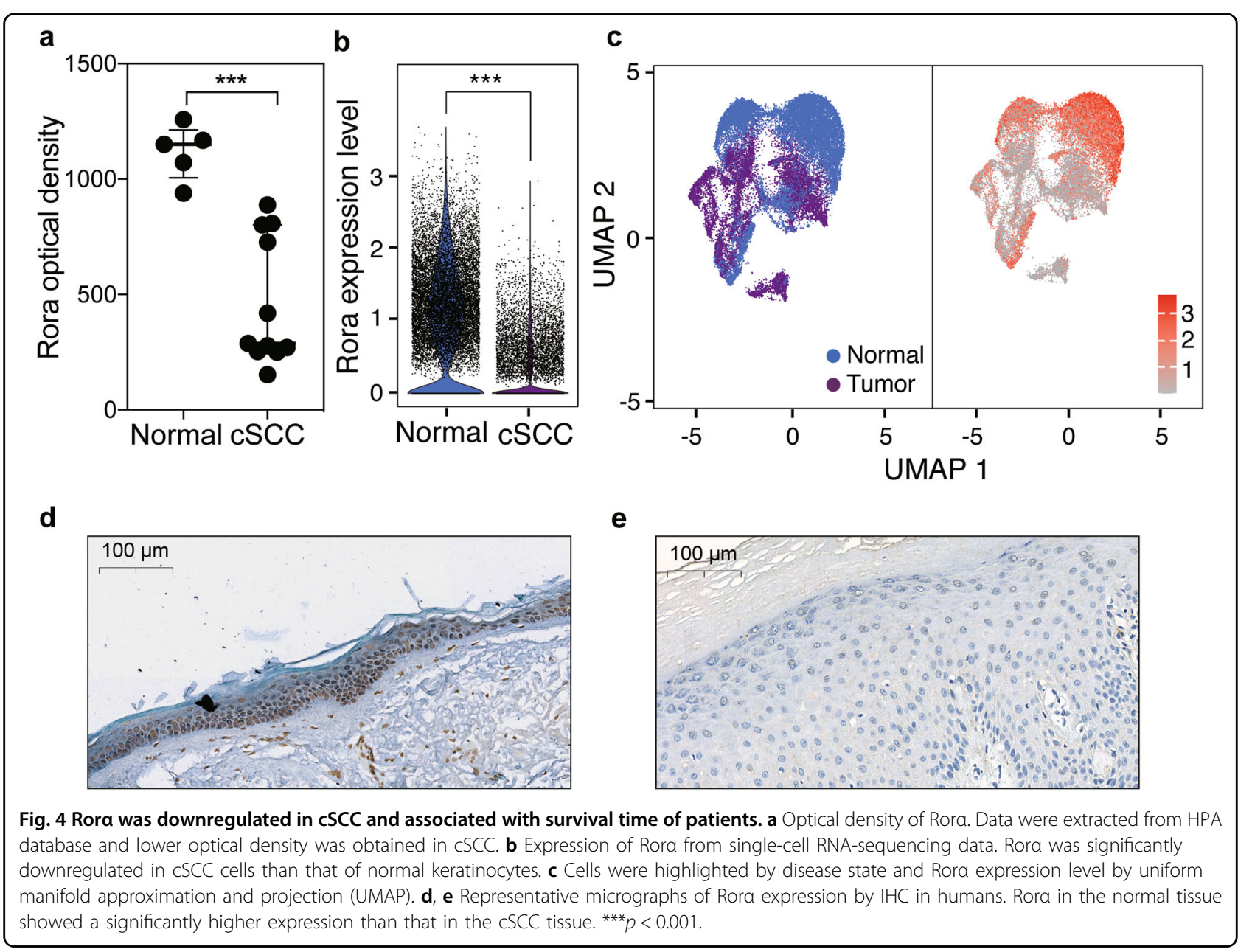

(HaCaT: 72 h, $p<0.01$; A431: 48 h, $p<0.01 ; 72$ h, $p<0.01$; PECA: $48 \mathrm{~h}, p<0.01,72 \mathrm{~h}, p<0.001$; Fig. $5 \mathrm{~b})$. By using siRNA interference with S100a9 and Sprr2f, no proliferation alteration of all three cells was seen (Fig. S5). In the wound-healing assay, all three cell lines showed significant migration inhibition after being stimulated by Ror $\alpha$ agonists (PECA: $p<0.01$ A A31: $p<0.01$; HaCaT: $p$ $<0.05)$. The cell migration of PECA $(P<0.05)$ and A431 $(p<0.05)$ was inhibited after siRNA interfered with S100a9, while there was a significant inhibitory effect after siRNA interferes with Sprr2f in PECA cell $(p<0.01)$ (Fig. $5 c, d)$. There was no effect on cell remote migration ability after up and downregulated the expressions of these three genes in terms of the transwell assay (Fig. S6). Finally, we employed fluorescent flow cytometry to reveal that the changed function of these three genes did not affect the apoptosis in both keratinocytes and cSCC cells (Fig. S7). In conclusion, activation of Ror $\alpha$ could downregulate the expressions of S100a9 and Sprr2f in cSCC cells, which play important role in cSCC progression. Furthermore, Ror $\alpha$ can inhibit cell proliferation and migration in cSCC cells, but not the normal keratinocyte.

\section{Rora activation inhibits CSCC growth}

To investigate whether Rord could affect the cSCC growth in vivo, we constructed a cSCC model by injecting XL50 cSCC cells subcutaneously into SKH-1 mice. Overexpressing Rora showed an inhibitory effect on tumorigenesis (Fig. 6a). Tumors in mice treated with Ror $\alpha$ agonist were significantly smaller than those of control mice (mean tumor volume: 86.82 vs. $204.77 \mathrm{~mm}^{3} ; p<$ 0.05; Fig. $6 \mathrm{~b}$ ). Then, tumors were weighed at the end of the experiment. Similarly, significantly lighter tumors were observed in the Ror $\alpha$ agonist group than the control group (57.28 vs. $132.96 \mathrm{mg} ; p<0.05$; Fig. $6 \mathrm{c}$ ). Finally, the isolated tumors were used to evaluate cell proliferation by IHC of Ki67 expression between SR1078 and the control group. The result showed that the proliferation of cSCC cells was attenuated by Ror $\alpha$ activation (Fig. 6d). Overall, Ror $\alpha$ is a critical regulator of tumor growth in CSCC, and the activation of Ror $\alpha$ can inhibit its growth.

\section{Discussion}

cSCC is quite common in melanoma skin cancer, and UV exposure including UVA and UVB was considered as 


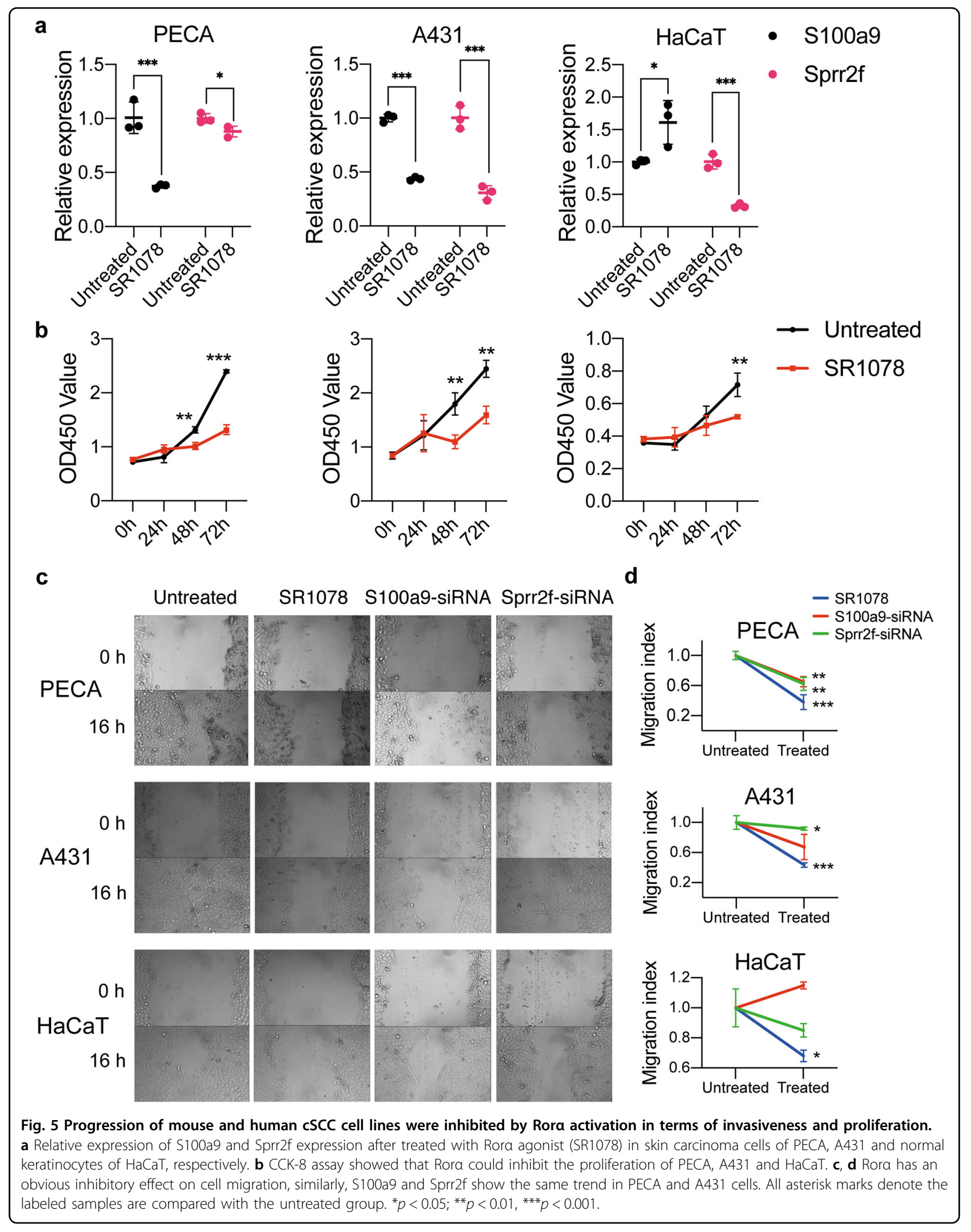


a

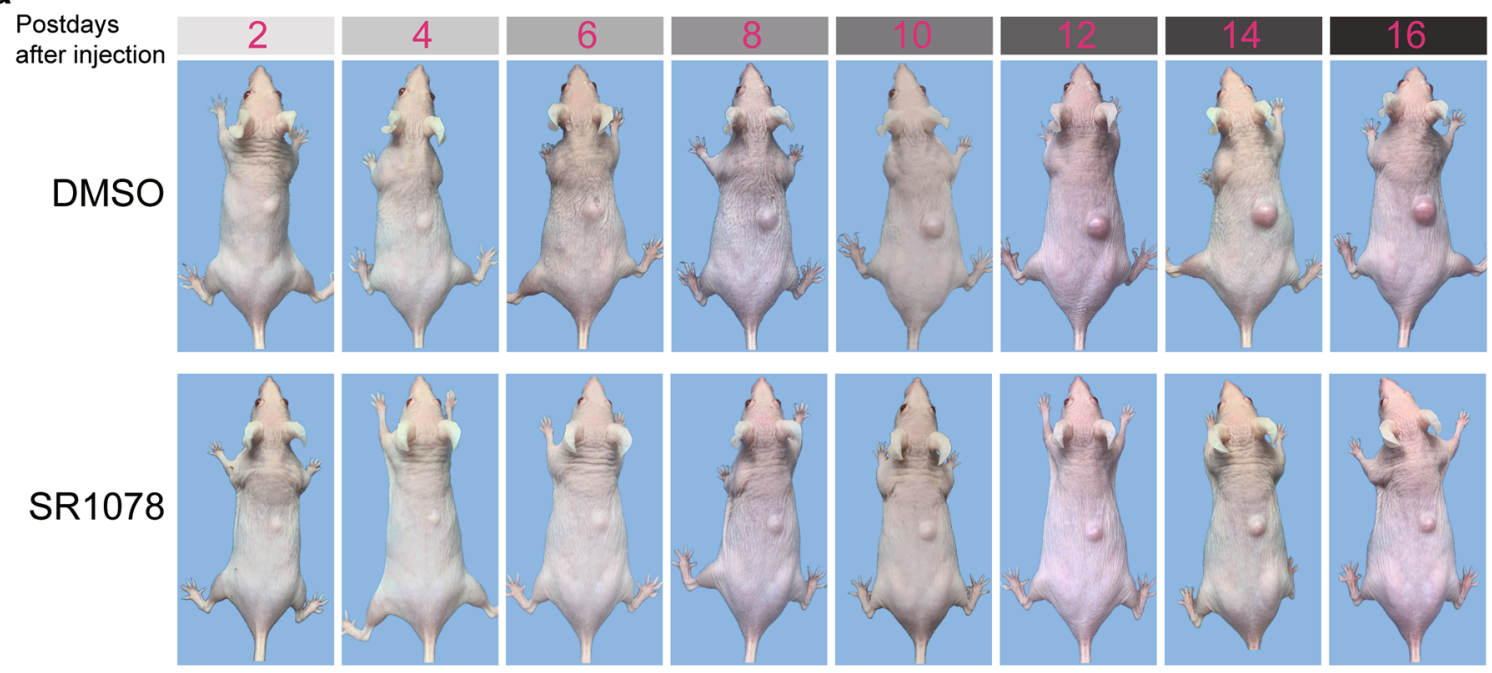

b

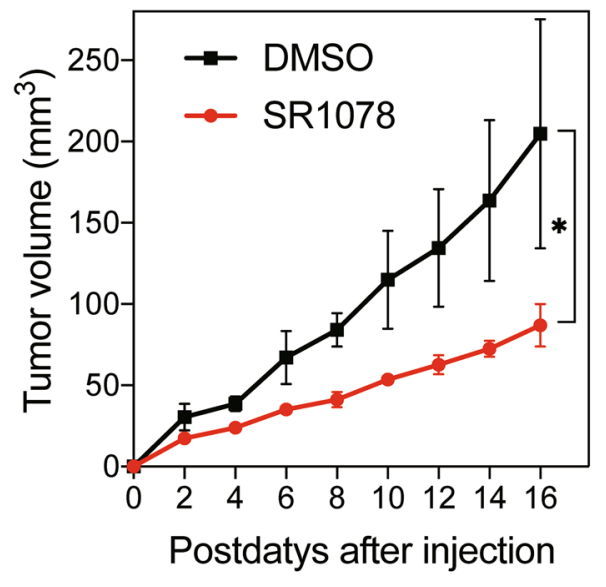

c

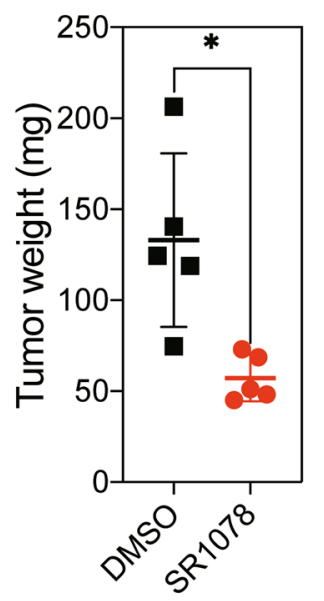

d
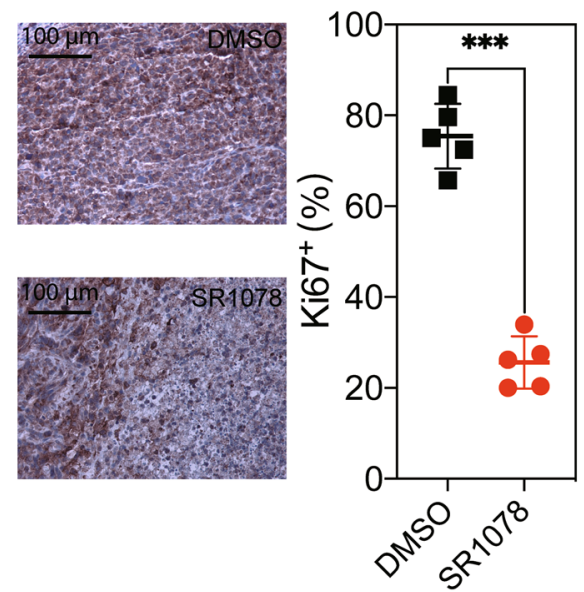

Fig. 6 Rora activation inhibits cSCC growth. a Representative tumors growth of SKH-1 mice after injected with cSCC cells between DMSO and SR1078 groups. b Tumor growth curve measured by tumor volume for DMSO and SR1078 groups. Tumor volumes were measured every two days and calculated as length $\times$ width ${ }^{2} \times 0.5\left(\mathrm{~mm}^{3}\right)$. c Tumor weight at sixteen days after injection with cSCC cells. Data are presented as the means \pm standard deviation. d Ki67 expression by IHC for DMSO and SR1078 groups. The proliferation of cSCC cells was attenuated by Rora activation. ${ }^{*} p<$ $0.05 ;{ }^{* *} p<0.01,{ }^{* * *} p<0.001$.

a main pathogenic factor. by overwhelming epidemiological, clinical, and biological data, it was thought that UV could cause considerable accumulated DNA damage and has been classified as a category 1a carcinogen by International Agency for the Research on Cancer (IARC) ${ }^{35}$. Study has demonstrated that there were many differences between UVA- and UVB-regulated miRNAs in human primary keratinocytes ${ }^{36}$, which indicates the unique detrimental function of both UVA and UVB. A recent study generated a CSCC mouse model only using UVB irradiation, and the UVB-regulated or the combination of UVAand UVB-regulated pathways involved in the CSCC progression may be ignored ${ }^{22}$. In the present study, we constructed a UV-induced cSCC mouse model covering the progression from NS to AK to cSCC, which mimicked the solar UV radiation perfectly using the solar-like ratio of UVA and UVB. The pathology of each stage was confirmed histologically.

A large number of DEGs were identified among NS, AK (the preneoplastic intermediate), and CSCC. Analyzing the complete gene expression data showed that AK held a similar mode with NS, regardless of the AK grades (Fig. 2a). This result was different from the study of Chitsazzadeh et al. ${ }^{22}$ that AK is more similar to CSCC with respect to the unsupervised clustering. This could be explained by the use of only UVB rather than the combination of UVA and UVB. Also, they only used the identified DEGs to generate the heatmap, which would 
further affect the clustering result. Although AK is similar to NS at a whole-transcriptome level, many identical DEGs were detected in AK and cSCC when compared with NS. Matrix metalloproteinase (Mmp) related genes including Mmp10 (stromelysin-2) and Mmp13 (collagenase-3) were detected in both $\mathrm{AK}$ and $\mathrm{cSCC}$, compared with NS. Moreover, Mmp8 and Mmp9 were also upregulated in cSCC. In specific, Mmp13 showed the highest fold change among Mmps in both AK and cSCC. It has been reported that Mmp10 and Mmp13 are highly expressed in tumor cells in $\mathrm{CSCC}^{37,38}$, and almost universally upregulated across all cancers by TCGA database analysis $^{39}$. The up-regulation of Mmps will degrade the extracellular matrix, which will promote cSCC cell invasion and implantation. Furthermore, the up-regulation of Mmps in AK implies that it may also promote the progression of AK to $\mathrm{CSCC}$.

Chemokines are essential coordinators for cell migration and interactions, which have a great impact on tumor development. Many chemokines were identified in cSCC and AK. In cSCC, $\mathrm{Cxcl} 2$ and $\mathrm{Cxcl} 3$ were identified, showing an angiogenic effect ${ }^{40}$. However, Cxcl9 and Cxcl10 were identified in AK, which exhibit an angiostatic effect $^{40}$. These results were determined by the malignant biological behavior in cSCC. When a mass of tumor cells struggle to rapidly proliferate, the tumor system requires accelerated neoangiogenesis to obtain more oxygen and nutrient, while no extra oxygen is required by AK. Inflammation is important in cancer. In the present study, inflammation-related chemokines including CCL3 and CCL4 were both increased in CSCC and AK, which helps recruit and $\mathrm{CD} 4^{+}, \mathrm{CD} 8^{+} \mathrm{T}$ cell to kill tumor cells ${ }^{41}$. A similar result was confirmed by GSVA analysis (Fig. 2b). Pathways of VEGF receptor signaling, hemoglobin biosynthesis, response to hypoxia, cofactor metabolism and mast cell chemotaxis and etc. were significantly unregulated in CSCC.

Although most studies considered that $\mathrm{AK}$ is a kind of precancerous lesion of cSCC, little study confirm the result at a transcriptome level. In the present study, a linear trajectory was re-constructed by transcriptome data. The progression trajectory clearly demonstrated that AK can progress into cSCC. From the genes involved in the pseudo-time trajectory, two TFs called Ror $\alpha$ together with Pou2f3 were sought out by co-expression analysis, which are regulatory factors for many genes including S100 family and Sprr family. Overexpression of Pou2f3 significantly inhibited the proliferation of $\mathrm{HaCaT}$ cells at 48 and $72 \mathrm{~h}$ after transfection ${ }^{42}$. In addition, Pou2f3 has been suggested as a tumor suppressor gene, which was downregulated in cervical cancer and melanoma ${ }^{43,44}$. Retinoid orphan nuclear receptors (RORs) are subfamily of the orphan nuclear receptors, which have been demonstrated to play vital roles in tumor progression and to be attractive therapeutic targets for many cancers ${ }^{45,46}$. Rord is one of the members of ROR subfamily and downregulated in many malignancies. In the present study, Ror $\alpha$ was predominantly downregulated in cSCC (Fig. 3d), and the low expression of Rora was confirmed by both scRNA-seq and solid biological experiments. We found that activation of Ror $\alpha$ inhibited cSCC cell proliferation and migration. In addition, we confirmed the anti-tumor effect of Rora activation in a cSCC mouse model in vivo. Extensively low expression of Ror $\alpha$ was noted in sixteen kind of cancers ${ }^{47}$. Furthermore, Ror $\alpha$ was proved to be responsible for the normal differentiation of keratinocyte, and showed a low expression in $\mathrm{CSCC}^{48}$. A positive correlation was also observed between the low expression of Rora and melanoma progression and short overall survival rate ${ }^{49,50}$, as well as unfavorable prognosis of breast cancer ${ }^{51}$, which can directly downregulate tumor suppressor gene Semaphorin 3F (Sema3f), causing tumor progression ${ }^{52}$. The extensively low expression of Ror $\alpha$ indicates an essential role in tumor progression.

Recent studies have suggested that the changed expression of S100 family may play a key step during cancer development and frequently observed in human epithelial tumors ${ }^{53}$. Here, we also noted that S100a9 was regulated by Rora and upregulated in CSCC (Fig. 3c). After being treated with Rora agonist in human and mouse cSCC cells, S100a9 expression was significantly downregulated, which confirmed the regulation effect of Ror $\alpha$. The further cell experiment confirmed the effect of proliferation and migration in cSCC cells by S100a9. S100a9 was also found to be upregulated in breast, lung, gastric, colorectal, pancreatic, and prostate cancer ${ }^{54}$, while downregulated in squamous oesophageal carcinomas ${ }^{55}$. Compared with extensive reports of S100a9, litter studies were published about Sprr2f. Sprr2f is a cross-linked envelope protein of keratinocyte, that first appears in the cell cytosol, but ultimately becomes cross-linked to membrane proteins by transglutaminase. Uniport data base showed that it is associated with cornification, epidermis development (https://www.uniprot.org/), the same function was proved by Takatori et al. ${ }^{56}$. A recent in silico analysis showed that Sprr2f was associated with ovarian carcinoma $^{57}$, which indicates that further study showed be conducted to elucidate the specific function of Sprr2f in tumor.

\section{Conclusion}

In summary, Ror $\alpha$ and Pou2f3 were identified to be significantly downregulated in CSCC by a UVR-induced cSCC mouse mode, which perfectly mimics the sunlight radiation in daily life. Solid bioinformatics analyses including scRNA-seq data and cell experiment together validated the expression of Ror $\alpha$. Moreover, the cell 
experiment confirmed the inhibition effect in cell proliferation and migration of Ror $\alpha$ by downregulating S100a9 and Sprr2f. Further anti-tumor effect of Rora activation was confirmed in a cSCC mouse model in vivo. Our findings demonstrated that Ror $\alpha$ would serve as a potential novel target for $\mathrm{CSCC}$, which will facilitate the treatment of $\mathrm{CSCC}$ in the future.

\section{Acknowledgements}

We acknowledged the nurses and the students involved in this project.

\section{Author details}

${ }^{1}$ Institute of Photomedicine, Shanghai Skin Disease Hospital, Tongji University School of Medicine, Shanghai 200443, China. ${ }^{2}$ State Key Laboratory of Genetic Engineering, School of Life Sciences, Fudan University, Shanghai 200438, China. ${ }^{3}$ Department of Epidemiology, School of Public Health, Fudan University, Shanghai 200438, China. ${ }^{4}$ Department of Pathology, Shanghai Skin Disease Hospital, Tongji University School of Medicine, Shanghai 200443, China

\section{Author contributions}

G.Z. designed the experiments and wrote the draft of the manuscript. G.Y. analyzed the data, wrote and revised the manuscript. Z.F. and X.B. analyzed part of the data and wrote part of the manuscript. Y.W., F.W., Z.Z., and S.F. collected the materials and conducted the experiments. Y.G. helped to manage and validate the data of the manuscript. Y.L., X.W., and S.Z. supervised this project and revised the manuscript. All authors read and approved the final manuscript.

\section{Funding}

This research was financially funded by the National Natural Science Foundation of China (81872212, 82073013, and 82073016), Shanghai Municipal Science and Technology Commission (19441904500).

\section{Data availability}

Raw data sets related to this study are available through the Gene Expression Omnibus (GSE158634).

\section{Ethics statement}

All procedures involved in mice complied with the protocol of the Animal Ethics Committee of Shanghai Skin Disease Hospital (No.2019-017).

\section{Conflict of interest}

The authors declare no competing interests.

\section{Publisher's note}

Springer Nature remains neutral with regard to jurisdictional claims in published maps and institutional affiliations.

Supplementary information The online version contains supplementary material available at https://doi.org/10.1038/s41419-021-03525-x.

Received: 17 October 2020 Revised: 9 February 2021 Accepted: 12 February 2021

Published online: 04 March 2021

\section{References}

1. Global Burden of Disease Cancer C, Fitzmaurice, C. et al. Global, regional, and national cancer incidence, mortality, years of life lost, years lived with disability, and disability-adjusted life-years for 29 cancer groups, 1990 to 2017: a systematic analysis for the global burden of disease study. JAMA Oncol. 5, 1749-1768 (2019)

2. Thompson, A. K., Kelley, B. F., Prokop, L. J., Murad, M. H. \& Baum, C. L. Risk factors for cutaneous squamous cell carcinoma recurrence, metastasis, and disease-specific death: a systematic review and meta-analysis. JAMA Dermatol. 152, 419-428 (2016).
3. Karia, P. S., Han, J. \& Schmults, C. D. Cutaneous squamous cell carcinoma: estimated incidence of disease, nodal metastasis, and deaths from disease in the United States, 2012. J. Am. Acad. Dermatol. 68, 957-966 (2013).

4. Roberts, M. R., Asgari, M. M. \& Toland, A. E. Genome-wide association studies and polygenic risk scores for skin cancer: clinically useful yet? Br. J. Dermatol. 181, 1146-1155 (2019).

5. Das Mahapatra, K. et al. A comprehensive analysis of coding and non-coding transcriptomic changes in cutaneous squamous cell carcinoma. Sci. Rep. 10, 3637 (2020).

6. Azimi, A. et al. Data independent acquisition proteomic analysis can discriminate between actinic keratosis, Bowen's disease, and cutaneous squamous cell carcinoma. J. Invest. Dermatol. 140, 212-222.e211 (2020).

7. Yao, Q. et al. Epigenetic alterations in keratinocyte carcinoma. J. Invest. Dermatol. 2020. (in press).

8. Siiskonen, S. J. et al. A genome-wide association study of cutaneous squamous cell carcinoma among European descendants. Cancer Epidemiol. Biomark. Prev. 25, 714-720 (2016).

9. Asgari, M. M. et al. Identification of susceptibility loci for cutaneous squamous cell carcinoma. J. Invest. Dermatol. 136, 930-937 (2016).

10. Chahal, H. S. et al. Genome-wide association study identifies novel susceptibility loci for cutaneous squamous cell carcinoma. Nat. Commun. 7, 12048 (2016).

11. South, A. P. et al. NOTCH1 mutations occur early during cutaneous squamous cell carcinogenesis. J. Invest. Dermatol. 134, 2630-2638 (2014).

12. Pickering, C. R. et al. Mutational landscape of aggressive cutaneous squamous cell carcinoma. Clin. Cancer Res. 20, 6582-6592 (2014).

13. Cammareri, P. et al. Inactivation of TGFbeta receptors in stem cells drives cutaneous squamous cell carcinoma. Nat. Commun. 7, 12493 (2016).

14. Inman, G. J. et al. The genomic landscape of cutaneous SCC reveals drivers and a novel azathioprine associated mutational signature. Nat. Commun. $\mathbf{9}$, 3667 (2018).

15. Garcia-Diez, I. et al. Transcriptome and cytogenetic profiling analysis of matched in situ/invasive cutaneous squamous cell carcinomas from immunocompetent patients. Genes Chromosomes Cancer 58, 164-174 (2019).

16. Li, L. et al. UVB induces cutaneous squamous cell carcinoma progression by de novo ID4 methylation via methylation regulating enzymes. EBioMedicine $\mathbf{5 7}$, 102835 (2020).

17. Hervas-Marin, D. et al. Genome wide DNA methylation profiling identifies specific epigenetic features in high-risk cutaneous squamous cell carcinoma. PLOS ONE 14, e0223341 (2019).

18. Siegel, J. A., Korgavkar, K. \& Weinstock, M. A. Current perspective on actinic keratosis: a review. Br. J. Dermatol. 177, 350-358 (2017).

19. Criscione, V. D. et al. Actinic keratoses: natural history and risk of malignant transformation in the Veterans Affairs Topical Tretinoin Chemoprevention Trial. Cancer 115, 2523-2530 (2009).

20. Durinck, S. et al. Temporal dissection of tumorigenesis in primary cancers. Cancer Discov. 1, 137-143 (2011).

21. Lambert, S. R. et al. Key differences identified between actinic keratosis and cutaneous squamous cell carcinoma by transcriptome profiling. Br. J. Cancer 110, 520-529 (2014).

22. Chitsazzadeh, V. et al. Cross-species identification of genomic drivers of squamous cell carcinoma development across preneoplastic intermediates. Nat. Commun. 7, 12601 (2016)

23. de Gruij, F. R. \& Forbes, P. D. UV-induced skin cancer in a hairless mouse model. Bioessays 17, 651-660 (1995).

24. Ananthaswamy, H. N. et al. p53 Mutations in hairless SKH-hr1 mouse skin tumors induced by a solar simulator. Photochem. Photobiol. 67, 2 (1998).

25. Lv, T. et al. Establishment of mouse model for cutaneous squamous cell carcinoma induced by ultraviolet. Chin. J. Dermatol. 44, 174-177 (2011).

26. Simon, A. FastQC: a quality control tool for high throughput sequence data. (2010).

27. Bolger, A. M., Lohse, M. \& Usadel, B. Trimmomatic: a flexible trimmer for Illumina sequence data. Bioinformatics 30, 2114-2120 (2014).

28. Pertea, M., Kim, D., Pertea, G. M., Leek, J. T. \& Salzberg, S. L. Transcript-leve expression analysis of RNA-seq experiments with HISAT, StringTie and Ballgown. Nat. Protoc. 11, 1650-1667 (2016).

29. Pertea, M. et al. StringTie enables improved reconstruction of a transcriptome from RNA-seq reads. Nat. Biotechnol. 33, 290-295 (2015).

30. Hanzelmann, S., Castelo, R. \& Guinney, J. GSVA: gene set variation analysis for microarray and RNA-seq data. BMC Bioinforma. 14, 7 (2013). 
31. Aran, D., Hu, Z. \& Butte, A. J. xCell: digitally portraying the tissue cellular heterogeneity landscape. Genome Biol. 18, 220 (2017).

32. Sturm, G. et al. Comprehensive evaluation of transcriptome-based cell-type quantification methods for immuno-oncology. Bioinformatics 35, i436-i445 (2019).

33. $\mathrm{Hu}, \mathrm{H}$. et al. AnimalTFDB 3.0: a comprehensive resource for annotation and prediction of animal transcription factors. Nucleic Acids Res. 47, D33-D38 (2019).

34. Davis, A. J. et al. TAp63-regulated miRNAs suppress cutaneous squamous cell carcinoma through inhibition of a network of Cell-cycle genes. Cancer Res. $\mathbf{8 0}$, 2484-2497 (2020)

35. El Ghissassi, F. et al. A review of human carcinogens-part D: radiation. Lancet Oncol. 10, 751-752 (2009).

36. Kraemer, A. et al. UVA and UVB irradiation differentially regulate microRNA expression in human primary keratinocytes. PLOS ONE 8, e83392 (2013).

37. Airola, K., Johansson, N., Kariniemi, A. L., Kahari, V. M. \& Saarialho-Kere, U. K. Human collagenase-3 is expressed in malignant squamous epithelium of the skin. J. Invest. Dermatol. 109, 225-231 (1997).

38. Ala-aho, R., Grenman, R., Seth, P. \& Kahari, V. M. Adenoviral delivery of p53 gene suppresses expression of collagenase-3 (MMP-13) in squamous carcinoma cells. Oncogene 21, 1187-1195 (2002).

39. Gobin, E. et al. A pan-cancer perspective of matrix metalloproteases (MMP) gene expression profile and their diagnostic/prognostic potential. BMC Cancer 19, 581 (2019).

40. Keeley, E. C., Mehrad, B. \& Strieter, R. M. CXC chemokines in cancer angiogenesis and metastases. Adv. Cancer Res. 106, 91-111 (2010).

41. Castellino, F. et al. Chemokines enhance immunity by guiding naive $C D 8^{+}$ T cells to sites of $\mathrm{CD4}^{+}$T cell-dendritic cell interaction. Nature 440, 890-895 (2006).

42. Ma, G. W. et al. Functional analysis of sheep POU2F3 isoforms. Biochem. Genet. 58, 335-347 (2020).

43. Zhang, Z. et al. Aberrant promoter methylation and silencing of the POU2F3 gene in cervical cancer. Oncogene 25, 5436-5445 (2006).

44. Riker, A. I. et al. The gene expression profiles of primary and metastatic melanoma yields a transition point of tumor progression and metastasis. BMC Med. Genomics 1, 13 (2008).
45. Vacca, M. et al. Nuclear receptors in regenerating liver and hepatocellular carcinoma. Mol. Cell. Endocrinol. 368, 108-119 (2013).

46. Huang, Q. et al. Retinoic acid-related orphan receptor $C$ isoform 2 expression and its prognostic significance for non-small cell lung cancer. J. Cancer Res. Clin. Oncol. 142, 263-272 (2016).

47. Parris, T. Z. Pan-cancer analyses of human nuclear receptors reveal transcriptome diversity and prognostic value across cancer types. Sci. Rep. 10, 1873 (2020).

48. Dai, J., Brooks, Y., Lefort, K., Getsios, S. \& Dotto, G. P. The retinoid-related orphan receptor RORalpha promotes keratinocyte differentiation via FOXN1. PLOS ONE 8, e70392 (2013).

49. Brozyna, A. A., Jozwicki, W., Roszkowski, K., Filipiak, J. \& Slominski, A. T. Melanin content in melanoma metastases affects the outcome of radiotherapy. Oncotarget 7, 17844-17853 (2016).

50. Slominski, A. T. et al. On the role of classical and novel forms of vitamin D in melanoma progression and management. J. Steroid Biochem. Mol. Biol. 177, 159-170 (2018).

51. Du, J. \& Xu, R. RORalpha, a potential tumor suppressor and therapeutic target of breast cancer. Int. J. Mol. Sci. 13, 15755-15766 (2012).

52. Xiong, G., Wang, C., Evers, B. M., Zhou, B. P. \& Xu, R. RORalpha suppresses breast tumor invasion by inducing SEMA3F expression. Cancer Res. 72, 1728-1739 (2012).

53. Gebhardt, C., Nemeth, J., Angel, P. \& Hess, J. S100A8 and S100A9 in inflammation and cancer. Biochem. Pharmacol. 72, 1622-1631 (2006).

54. Cross, S. S., Hamdy, F. C., Deloulme, J. C. \& Rehman, I. Expression of S100 proteins in normal human tissues and common cancers using tissue microarrays: S100A6, S100A8, S100A9 and S100A11 are all overexpressed in common cancers. Histopathology 46, 256-269 (2005).

55. Luo, A. et al. Discovery of $\mathrm{Ca}_{2}^{+}{ }^{+}$-relevant and differentiation-associated genes downregulated in esophageal squamous cell carcinoma using cDNA microarray. Oncogene 23, 1291-1299 (2004).

56. Takatori, R. et al. Effects of oral administration of glucosylceramide on gene expression changes in hairless mouse skin: comparison of whole skin, epidermis, and dermis. Biosci. Biotechnol. Biochem. 77, 1882-1887 (2013).

57. Wang, Y., Lei, L., Chi, Y. G., Liu, L. B. \& Yang, B. P. A comprehensive understanding of ovarian carcinoma survival prognosis by novel biomarkers. Eur. Rev. Med. Pharmacol. Sci. 23, 8257-8264 (2019). 